\title{
Home parenteral nutrition: the influence of domestic, behavioural and procedural factors on catheter-related bloodstream infection
}

\author{
D. Jones ${ }^{1,2}$, A. Davidson ${ }^{2}$, Y. M. Jeanes ${ }^{1}$, K. Sharp ${ }^{1}$ and S. M. Gabe ${ }^{2}$ \\ ${ }^{1}$ School of Human and Life Sciences, Roehampton University, London SW15 4JD, UK and ${ }^{2}$ Lennard-Jones Intestinal \\ Failure Unit, St Mark's Hospital, NWLH NHS Trust HA1 3UJ, UK
}

Catheter-related bloodstream infection (CRBSI) is the most frequent complication in home parenteral nutrition (HPN) ${ }^{(1)}$. Investigations on the potential influence of the home environment on the incidence of CRBSI in patients receiving HPN are small and few. The aim of the present study was to evaluate a range of factors in the home environment that may influence the incidence of CRBSI in patients on HPN.

In a retrospective study of fifty patients receiving HPN from a tertiary centre for intestinal failure, data on domestic, behavioural and procedural variables were collected during a one-to-one semi-structured interview. Data on CRBSI episodes including causative microbial agent(s) were obtained from patient medical records. Inclusion criteria included: on HPN (including fluid and electrolytes only) between January 2004 and June 2005; aged > 18 years; HPN administered by themselves or by a specialist HPN nursing team.

A total of $84 \%$ of participants had experienced one or more CRBSI since commencing HPN (mean duration of HPN 7.4 years). In total $42 \%$ were diagnosed with one or more CRBSI during the 18-month study period, resulting in an incidence of CRBSI of 6.2 per $100 \mathrm{~d}$ of HPN. The majority of the sample ( $88 \%$ ) performed their HPN procedures in two of three main rooms (bathroom, kitchen, bedroom) in the home. Only six participants (12\%) remained in the same room for the entire procedure. Frequency of infusions, procedural short cuts and consumption of medication with sedative properties were not associated with CRBSI $(P<0.2)$. Inclusion of glucose and/or lipid energy $(P=0.05)$ and access of pets to the room(s) in which the HPN procedures were performed $(P=0.04)$ were found to significantly increase the risk of CRBSI. Nineteen of the participants (38\%) shared their home with one or more pets and, of these, seven participants (37\%) did not allow their pet(s) into their HPN procedure room(s) at any time.

Factors in the home environment appear to play a role in CRBSI. Interestingly, having one or more pets in the home environment does not appear to have any impact on the incidence of CRBSI; however, the present study suggests that allowing pets into the procedure room(s) is one of the most significant risk factors for CRBSI. In addition, the current study did not demonstrate any increased risk of CRBSI with opiate use, contrary to a previous study ${ }^{(2)}$. A larger study is required to confirm these results. Comparison between studies in this area is compromised by the lack of universal guidelines on the definition and diagnostic criteria of CRBSI.

1. Reimund J-M, Arondel Y, Finck G et al. (2002) Clin Nutr 21, 33-38.

2. Richards DM, Scott NA, Shaffer JL et al. (1997) J Parenter Enteral Nutr 21, 336-338. 